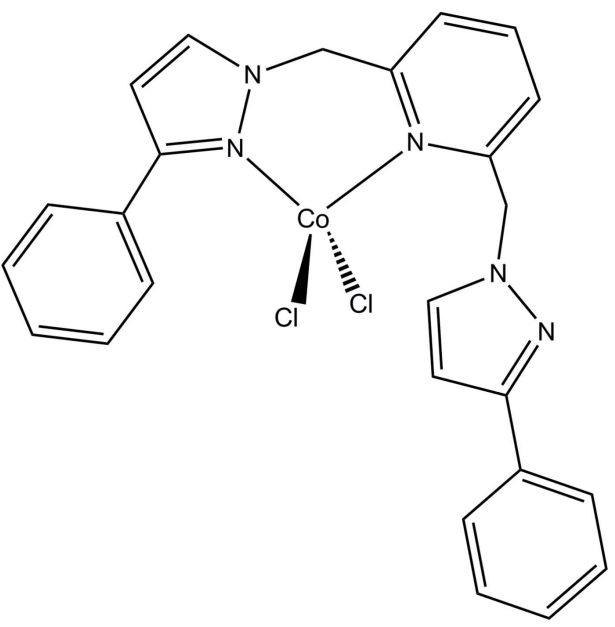

\title{
Crystal structure of dichlorido\{2,6-bis[(3- phenyl-1H-pyrazol-1-yl)methyl]- pyridine $\}_{\text {cobalt(II) }}$
}

\section{Kyung-sun Son, Jeong Oh Woo, Daeyoung Kim and Sung Kwon Kang*}

Department of Chemistry, Chungnam National University, Daejeon 305-764, Republic of Korea. *Correspondence e-mail: skkang@cnu.ac.kr

Received 20 February 2015; accepted 24 February 2015

Edited by E. R. T. Tiekink, University of Malaya, Malaysia

In the title complex, $\left[\mathrm{CoCl}_{2}\left(\mathrm{C}_{25} \mathrm{H}_{21} \mathrm{~N}_{5}\right)\right]$, the $\mathrm{Co}^{\mathrm{II}}$ atom is coordinated by two $\mathrm{Cl}$ atoms and two $\mathrm{N}$ atoms, provided by a tridentate pyrazolylpyridyl ligand, forming a slightly distorted tetrahedral geometry [range of angles: 96.51 (10) (chelate ring) to $118.60(9)^{\circ}$. The dihedral angle between $\mathrm{Cl} / \mathrm{Co} / \mathrm{Cl}$ and $\mathrm{N} / \mathrm{Co} / \mathrm{N}$ planes is $86.83(7)^{\circ}$. The chelate ring has the conformation of a distorted boat. The dihedral angle between pyridyl ring and the coordinated pyrazolyl ring is $56.16(12)^{\circ}$. The uncoordinated pyrazolyl ring is almost perpendicular to the pyridyl ring with the dihedral angle of $87.49(10)^{\circ}$. In the crystal packing, intermolecular phenyl-C $-\mathrm{H} \cdots \pi$ (pyridyl) interactions generate dimeric aggregates. These are connected into a zigzag supramolecular chain along the $c$-axis direction via $\pi-\pi$ interactions [inter-centroid distance between pyridyl and phenyl rings $=3.664(2) \AA]$.

Keywords: crystal structure; Co" complex; pyrazolylpyridyl; $\mathrm{C}-\mathrm{H} \cdots \pi$ interactions; $\pi-\pi$ interactions.

CCDC reference: 1051138

\section{Related literature}

For the synthesis of the title compound, see: Reger et al. (2005); Son et al. (2014). For metal complexes with similar ligands, see: Massoud et al. (2013); Sharma et al. (2011); Ojwach et al. (2007); Manikandan et al. (2000, 2001); Halcrow \& Kilner (2002); Foster et al. (2002). For the potential applications of the ligand in catalysis, see: Karam et al. (2005).

\section{Experimental}

\subsection{Crystal data}

$\left[\mathrm{CoCl}_{2}\left(\mathrm{C}_{25} \mathrm{H}_{21} \mathrm{~N}_{5}\right)\right]$

$M_{r}=521.3$

Monoclinic, $C 2 / c$

$a=12.9766$ (4) $\AA$

$b=10.5867$ (3) $\AA$

$c=33.5943(9) \AA$

$\beta=93.2592(19)^{\circ}$

$V=4607.7(2) \AA^{3}$

$Z=8$

Mo $K \alpha$ radiation

$\mu=1.00 \mathrm{~mm}^{-1}$

$T=296 \mathrm{~K}$

$0.25 \times 0.22 \times 0.1 \mathrm{~mm}$

\subsection{Data collection}

Bruker SMART CCD area-detector diffractometer

Absorption correction: multi-scan (SADABS; Bruker, 2002)

$T_{\min }=0.76, T_{\max }=0.903$

25697 measured reflections 5718 independent reflections 3751 reflections with $I>2 \sigma(I)$ $R_{\text {int }}=0.072$

\subsection{Refinement}

$R\left[F^{2}>2 \sigma\left(F^{2}\right)\right]=0.058$

$w R\left(F^{2}\right)=0.132$

$S=1.06$

5718 reflections

298 parameters

$\mathrm{H}$-atom parameters constrained

$\Delta \rho_{\max }=0.52{\mathrm{e} \AA^{-3}}^{-3}$

$\Delta \rho_{\min }=-0.47$ e $\AA^{-3}$

Table 1

Selected bond lengths $(\AA)$.

\begin{tabular}{llll}
\hline $\mathrm{Co} 1-\mathrm{N} 4$ & $2.041(3)$ & $\mathrm{Co} 1-\mathrm{Cl} 3$ & $2.2030(11)$ \\
$\mathrm{Co} 1-\mathrm{N} 17$ & $2.109(3)$ & $\mathrm{Co} 1-\mathrm{Cl} 2$ & $2.2499(10)$ \\
\hline
\end{tabular}

Table 2

Hydrogen-bond geometry $\left(\AA{ }^{\circ}\right)$.

\begin{tabular}{lllll}
\hline$D-\mathrm{H} \cdots A$ & $D-\mathrm{H}$ & $\mathrm{H} \cdots A$ & $D \cdots A$ & $D-\mathrm{H} \cdots A$ \\
\hline $\mathrm{C} 11-\mathrm{H} 11 \cdots C g 1^{\mathrm{i}}$ & 0.93 & 2.87 & $3.806(4)$ & 180 \\
\hline
\end{tabular}

Symmetry code: (i) $-x+1,-y,-z+1$.

Data collection: SMART (Bruker, 2002); cell refinement: SAINT (Bruker, 2002); data reduction: $S A I N T$; $\operatorname{program}(\mathrm{s})$ used to solve structure: SHELXS2013 (Sheldrick, 2008); program(s) used to refine 
structure: SHELXL2013 (Sheldrick, 2015); molecular graphics: ORTEP-3 for Windows (Farrugia, 2012); software used to prepare material for publication: WinGX (Farrugia, 2012).

\section{Acknowledgements}

Financial support from the National Research Foundation of Korea (NRF-2012R1A1A1005839) is gratefully acknowledged.

Supporting information for this paper is available from the IUCr electronic archives (Reference: TK5360).

\section{References}

Bruker (2002). SADABS, SAINT and SMART. Bruker AXS Inc., Madison, Wisconsin, USA.

Farrugia, L. J. (2012). J. Appl. Cryst. 45, 849-854.
Foster, C. L., Kilner, C. A., Thornton-Pett, M. \& Halcrow, M. A. (2002). Polyhedron, 21, 1031-1041.

Halcrow, M. A. \& Kilner, C. A. (2002). Acta Cryst. C58, m424-m426.

Karam, A. R., Catarí, E. L., López-Linares, F., Agrifoglio, G., Albano, C. L., Díaz-Barrios, A., Lehmann, T. E., Pekerar, S. V., Albornoz, L. A., Atencio, R., González, T., Ortega, H. B. \& Joskowics, P. (2005). Appl. Catal. A, 280, 165-173.

Manikandan, P., Justin Thomas, K. R. \& Manoharan, P. T. (2000). J. Chem. Soc. Dalton Trans. pp. 2779-2785.

Manikandan, P., Padmakumar, K., Justin Thomas, K. R., Varghese, B., Onodera, H. \& Manoharan, P. T. (2001). Inorg. Chem. 40, 6930-6939.

Massoud, S. S., Guilbeau, A. E., Luong, H. T., Vicente, R., Albering, J. H., Fischer, R. C. \& Mautner, F. A. (2013). Polyhedron, 54, 26-33.

Ojwach, S. O., Guzei, I. A., Darkwa, J. \& Mapolie, S. F. (2007). Polyhedron, 26, 851-861.

Reger, D. L., Semeniuc, R. F. \& Smith, M. D. (2005). Cryst. Growth Des. 5, $1181-1190$.

Sharma, A. K., De, A., Balamurugan, V. \& Mukherjee, R. (2011). Inorg. Chim. Acta, 372, 327-332.

Sheldrick, G. M. (2008). Acta Cryst. A64, 112-122.

Sheldrick, G. M. (2015). Acta Cryst. C71, 3-8.

Son, K., Woo, J. O., Kim, D. \& Kang, S. K. (2014). Acta Cryst. E70, o973. 


\title{
supporting information
}

Acta Cryst. (2015). E71, m75-m76 [doi:10.1107/S2056989015003862]

\section{Crystal structure of dichlorido\{2,6-bis[(3-phenyl-1H-pyrazol-1-yl)methyl]- pyridine\}cobalt(II)}

\author{
Kyung-sun Son, Jeong Oh Woo, Daeyoung Kim and Sung Kwon Kang
}

\section{S1. Experimental}

To a stirred solution of 2,6-pyridinedimethanol $(0.28 \mathrm{~g}, 2.0 \mathrm{mmol})$ and $\mathrm{NaOH}(0.8 \mathrm{~g}, 20 \mathrm{mmol})$ in tetrahydrofuran $(\mathrm{THF}) /$ water $(7.5 / 7.5 \mathrm{ml})$ was added a solution of $p$-toluenesulfonyl chloride $(0.76 \mathrm{~g}, 4.0 \mathrm{mmol})$ in THF $(7.5 \mathrm{ml})$ at $0{ }^{\circ} \mathrm{C}$. After $4 \mathrm{~h}$ of stirring, the mixture was poured into $20 \mathrm{ml}$ of water and extracted with methylene chloride three times. The organic layer was washed with saturated aqueous $\mathrm{NaCl}$ solution and distilled water, and dried over $\mathrm{Na}_{2} \mathrm{SO}_{4}$; the solvent was removed in vacuo to afford 2,6-pyridine-dimethylene-ditosylate $(0.788 \mathrm{~g}, 88 \%)$ as a white powder. In a separate flask under a nitrogen atmosphere, a solution of 3-phenyl- $1 H$-pyrazole $(0.61 \mathrm{~g}, 5.34 \mathrm{mmol})$ in dry THF $(10 \mathrm{ml})$ was added drop-wise to a suspension of $\mathrm{NaH}(0.13 \mathrm{~g}, 5.34 \mathrm{mmol})$ in dry THF $(10 \mathrm{ml})$ at $0{ }^{\circ} \mathrm{C}$. After $15 \mathrm{~min}$ of stirring, a solution of 2,6-pyridine-dimethylene-ditosylate $(1.20 \mathrm{~g}, 2.67 \mathrm{mmol})$ in dry THF $(15 \mathrm{ml})$ was added to this solution; the mixture was stirred overnight, filtered, and the solvent was removed. The crude product was purified by column chromatography on silica gel with ethyl acetate : hexane $=1: 1$ as eluent to afford $0.41 \mathrm{~g}(40 \%)$ of pure ligand as a white oil.

To a solution of $\mathrm{CoCl}_{2}(2.6 \mathrm{mg}, 0.02 \mathrm{mmol})$ in THF $(2 \mathrm{ml})$ was added a solution of the organic ligand $(7.8 \mathrm{mg}, 0.02$ $\mathrm{mmol})$ in THF $(2 \mathrm{ml})$ drop-wise at $40{ }^{\circ} \mathrm{C}$. The solution was stirred vigorously. A deep-blue suspension was formed immediately. The product was isolated as a blue powder by removing the solvent, washed repeatedly with THF followed by diethyl ether, and dried in vacuo. Deep-blue single crystals of the title compound were obtained by slow evaporation of its concentrated solution in dichloromethane at room temperature.

\section{S2. Refinement}

All $\mathrm{H}$ atoms were positioned geometrically and refined using a riding model, with $\mathrm{C}-\mathrm{H}=0.93-0.97 \AA$, and with $\mathrm{U}_{\text {iso }}(\mathrm{H})$ $=1.2 \mathrm{U}_{\mathrm{eq}}(\mathrm{C})$. 


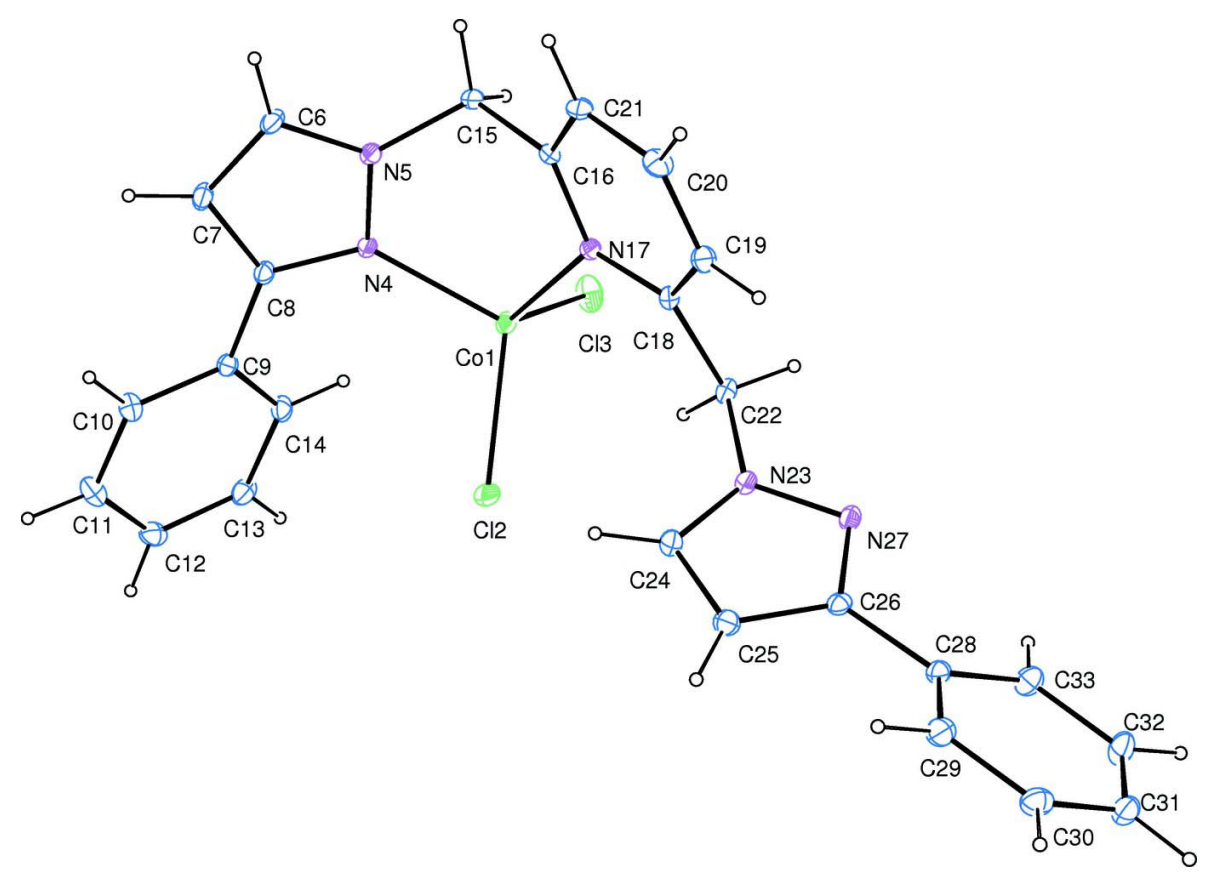

Figure 1

Molecular structure of the title compound, showing the atom-numbering scheme and 30\% probability ellipsoids.

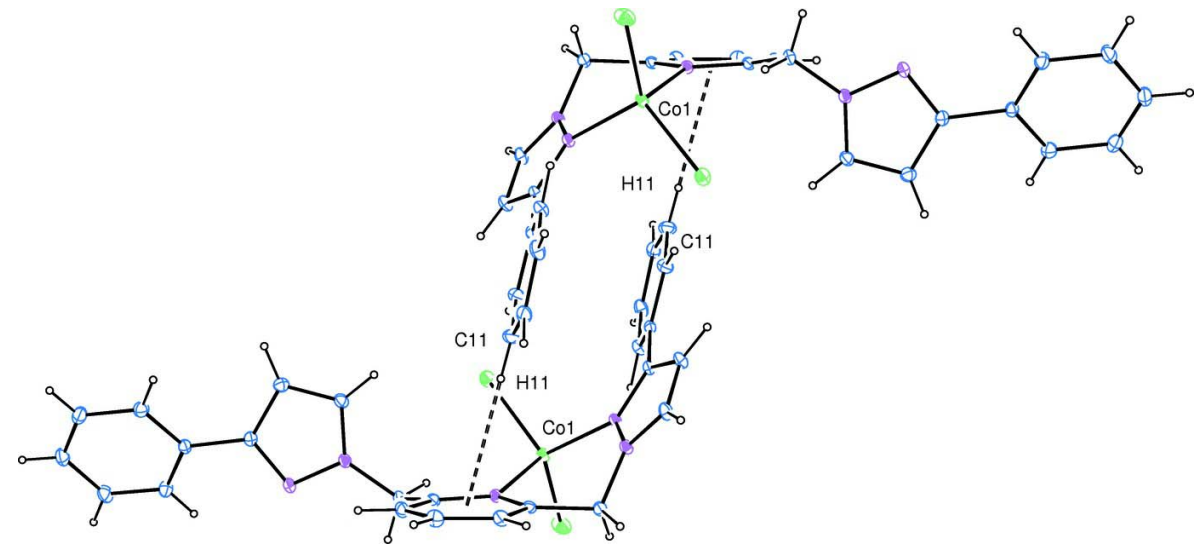

Figure 2

Dimer formation via $\mathrm{C}-\mathrm{H} \cdots \pi$ interactions.

Dichlorido\{2,6-bis[(3-phenyl-1H-pyrazol-1-yl)methyl]pyridine\}cobalt(II)

\section{Crystal data}

$\left[\mathrm{CoCl}_{2}\left(\mathrm{C}_{25} \mathrm{H}_{21} \mathrm{~N}_{5}\right)\right]$

$M_{r}=521.3$

Monoclinic, $C 2 / c$

Hall symbol: $-\mathrm{C} 2 \mathrm{yc}$

$a=12.9766$ (4) $\AA$

$b=10.5867$ (3) $\AA$

$c=33.5943(9) \AA$

$\beta=93.2592(19)^{\circ}$

$V=4607.7(2) \AA^{3}$

$Z=8$
$F(000)=2136$

$D_{\mathrm{x}}=1.503 \mathrm{Mg} \mathrm{m}^{-3}$

Mo $K \alpha$ radiation, $\lambda=0.71073 \AA$

Cell parameters from 4379 reflections

$\theta=2.4-23.4^{\circ}$

$\mu=1.00 \mathrm{~mm}^{-1}$

$T=296 \mathrm{~K}$

Plate, deep-blue

$0.25 \times 0.22 \times 0.1 \mathrm{~mm}$ 


\section{Data collection}

Bruker SMART CCD area-detector diffractometer

Radiation source: fine-focus sealed tube $\varphi$ and $\omega$ scans

Absorption correction: multi-scan (SADABS; Bruker, 2002)

$T_{\min }=0.76, T_{\max }=0.903$

25697 measured reflections

\section{Refinement}

Refinement on $F^{2}$

Least-squares matrix: full

$R\left[F^{2}>2 \sigma\left(F^{2}\right)\right]=0.058$

$w R\left(F^{2}\right)=0.132$

$S=1.06$

5718 reflections

298 parameters

0 restraints
5718 independent reflections

3751 reflections with $I>2 \sigma(I)$

$R_{\text {int }}=0.072$

$\theta_{\max }=28.3^{\circ}, \theta_{\min }=2.4^{\circ}$

$h=-15 \rightarrow 17$

$k=-14 \rightarrow 14$

$l=-44 \rightarrow 44$

Hydrogen site location: inferred from neighbouring sites

$\mathrm{H}$-atom parameters constrained

$w=1 /\left[\sigma^{2}\left(F_{\mathrm{o}}^{2}\right)+(0.048 P)^{2}+4.3485 P\right]$

where $P=\left(F_{\mathrm{o}}{ }^{2}+2 F_{\mathrm{c}}{ }^{2}\right) / 3$

$(\Delta / \sigma)_{\max }=0.002$

$\Delta \rho_{\max }=0.52 \mathrm{e} \AA^{-3}$

$\Delta \rho_{\min }=-0.47$ e $\AA^{-3}$

\section{Special details}

Geometry. All e.s.d.'s (except the e.s.d. in the dihedral angle between two 1.s. planes) are estimated using the full covariance matrix. The cell e.s.d.'s are taken into account individually in the estimation of e.s.d.'s in distances, angles and torsion angles; correlations between e.s.d.'s in cell parameters are only used when they are defined by crystal symmetry. An approximate (isotropic) treatment of cell e.s.d.'s is used for estimating e.s.d.'s involving 1.s. planes.

Fractional atomic coordinates and isotropic or equivalent isotropic displacement parameters $\left(\AA^{2}\right)$

\begin{tabular}{|c|c|c|c|c|}
\hline & $x$ & $y$ & $z$ & $U_{\text {iso }} * / U_{\text {eq }}$ \\
\hline Col & $0.39083(3)$ & 0.22404 & $0.58917(2)$ & $0.03468(14)$ \\
\hline $\mathrm{Cl} 2$ & $0.56212(7)$ & $0.23374(10)$ & 0.58231 & $0.0500(2)$ \\
\hline $\mathrm{Cl} 3$ & $0.32167(10)$ & $0.40748(10)$ & 0.60338 & $0.0672(3)$ \\
\hline N4 & $0.3229(2)$ & $0.1187(3)$ & $0.54406(7)$ & $0.0331(6)$ \\
\hline N5 & $0.2543(2)$ & 0.0299 & $0.55511(7)$ & $0.0366(6)$ \\
\hline C6 & 0.2331 & $-0.0533(3)$ & $0.52567(10)$ & $0.0443(9)$ \\
\hline H6 & 0.1887 & -0.122 & 0.5266 & $0.053 *$ \\
\hline $\mathrm{C} 7$ & 0.2884 & -0.0185 & $0.49419(10)$ & $0.0436(9)$ \\
\hline $\mathrm{H} 7$ & 0.2894 & -0.059 & 0.4696 & $0.052 *$ \\
\hline $\mathrm{C} 8$ & 0.3435 & $0.0901(3)$ & $0.50604(9)$ & $0.0347(7)$ \\
\hline C9 & $0.4137(2)$ & $0.1637(3)$ & $0.48268(9)$ & $0.0342(7)$ \\
\hline $\mathrm{C} 10$ & $0.4601(3)$ & $0.1057(4)$ & $0.45118(10)$ & $0.0475(9)$ \\
\hline $\mathrm{H} 10$ & 0.4465 & 0.021 & 0.4457 & $0.057 *$ \\
\hline $\mathrm{C} 11$ & $0.5260(3)$ & $0.1714(4)$ & $0.42791(12)$ & $0.0562(11)$ \\
\hline H11 & 0.5554 & 0.1316 & 0.4066 & $0.067 *$ \\
\hline $\mathrm{C} 12$ & 0.5480 & $0.2960(4)$ & $0.43629(11)$ & $0.0534(11)$ \\
\hline $\mathrm{H} 12$ & 0.5953 & 0.3394 & 0.4216 & $0.064 *$ \\
\hline $\mathrm{C} 13$ & 0.5003 & $0.3558(4)$ & $0.46622(11)$ & $0.0509(10)$ \\
\hline $\mathrm{H} 13$ & 0.513 & 0.4411 & 0.471 & $0.061 *$ \\
\hline $\mathrm{C} 14$ & 0.4333 & $0.2909(4)$ & $0.48940(10)$ & $0.0436(9)$ \\
\hline $\mathrm{H} 14$ & 0.4012 & 0.3328 & 0.5096 & $0.052 *$ \\
\hline $\mathrm{C} 15$ & 0.2098 & $0.0399(4)$ & $0.59390(9)$ & $0.0403(8)$ \\
\hline
\end{tabular}




\begin{tabular}{|c|c|c|c|c|}
\hline H15A & 0.1516 & -0.0174 & 0.5947 & $0.048^{*}$ \\
\hline H15B & 0.1841 & 0.1251 & 0.5972 & $0.048^{*}$ \\
\hline $\mathrm{C} 16$ & $0.2864(2)$ & 0.0096 & $0.62810(9)$ & $0.0333(7)$ \\
\hline N17 & $0.3619(2)$ & $0.0971(2)$ & $0.63560(7)$ & $0.0314(6)$ \\
\hline $\mathrm{C} 18$ & $0.4258(2)$ & $0.0796(3)$ & $0.66823(9)$ & $0.0330(7)$ \\
\hline C19 & $0.4218(3)$ & $-0.0281(4)$ & $0.69132(10)$ & $0.0430(9)$ \\
\hline H19 & 0.4689 & -0.0395 & 0.7129 & $0.052 *$ \\
\hline $\mathrm{C} 20$ & $0.3486(3)$ & $-0.1176(4)$ & $0.68232(11)$ & $0.0492(10)$ \\
\hline $\mathrm{H} 20$ & 0.346 & -0.1911 & 0.6974 & $0.059^{*}$ \\
\hline $\mathrm{C} 21$ & $0.2784(3)$ & -0.0975 & $0.65052(10)$ & $0.0438(9)$ \\
\hline $\mathrm{H} 21$ & 0.2264 & -0.156 & 0.6444 & $0.053^{*}$ \\
\hline $\mathrm{C} 22$ & $0.4977(3)$ & $0.1871(3)$ & $0.68058(10)$ & $0.0364(8)$ \\
\hline $\mathrm{H} 22 \mathrm{~A}$ & 0.5015 & 0.2456 & 0.6585 & $0.044 *$ \\
\hline $\mathrm{H} 22 \mathrm{~B}$ & 0.4696 & 0.2324 & 0.7026 & $0.044 *$ \\
\hline $\mathrm{N} 23$ & $0.6008(2)$ & 0.1435 & $0.69249(8)$ & $0.0363(6)$ \\
\hline $\mathrm{C} 24$ & $0.6654(3)$ & 0.0790 & $0.67056(10)$ & $0.0420(8)$ \\
\hline $\mathrm{H} 24$ & 0.6533 & 0.0544 & 0.6441 & $0.05^{*}$ \\
\hline $\mathrm{C} 25$ & $0.7519(3)$ & 0.0558 & $0.69398(10)$ & $0.0436(9)$ \\
\hline $\mathrm{H} 25$ & 0.8105 & 0.0122 & 0.6871 & $0.052 *$ \\
\hline $\mathrm{C} 26$ & $0.7342(3)$ & $0.1112(3)$ & $0.73064(9)$ & $0.0352(7)$ \\
\hline $\mathrm{N} 27$ & $0.6417(2)$ & $0.1659(3)$ & $0.72992(8)$ & $0.0374(7)$ \\
\hline $\mathrm{C} 28$ & $0.8065(3)$ & $0.1190(3)$ & $0.76634(10)$ & $0.0376(8)$ \\
\hline $\mathrm{C} 29$ & $0.8786(3)$ & 0.0243 & $0.77423(12)$ & $0.0503(9)$ \\
\hline $\mathrm{H} 29$ & 0.8804 & -0.0454 & 0.7574 & $0.06^{*}$ \\
\hline $\mathrm{C} 30$ & $0.9484(3)$ & $0.0329(4)$ & $0.80719(12)$ & $0.0550(10)$ \\
\hline $\mathrm{H} 30$ & 0.9961 & -0.0314 & 0.8124 & $0.066^{*}$ \\
\hline $\mathrm{C} 31$ & $0.9473(3)$ & $0.1347(4)$ & 0.83167 (11) & $0.0557(11)$ \\
\hline H31 & 0.9954 & 0.1413 & 0.8532 & $0.067^{*}$ \\
\hline C32 & $0.8750(3)$ & $0.2281(4)$ & $0.82467(12)$ & $0.0586(11)$ \\
\hline $\mathrm{H} 32$ & 0.873 & 0.2968 & 0.8419 & $0.07^{*}$ \\
\hline $\mathrm{C} 33$ & $0.8047(3)$ & $0.2202(4)$ & 0.79195 (11) & $0.0493(9)$ \\
\hline $\mathrm{H} 33$ & 0.756 & 0.2838 & 0.7874 & $0.059 *$ \\
\hline
\end{tabular}

Atomic displacement parameters $\left(\AA^{2}\right)$

\begin{tabular}{lllllll}
\hline & $U^{11}$ & $U^{22}$ & $U^{33}$ & $U^{12}$ & $U^{13}$ & $U^{23}$ \\
\hline Co1 & $0.0414(3)$ & $0.0335(3)$ & $0.0290(2)$ & $-0.0090(2)$ & $0.00033(17)$ & $-0.00083(19)$ \\
C12 & $0.0439(5)$ & $0.0617(6)$ & $0.0444(5)$ & $-0.0201(5)$ & $0.0022(4)$ & $0.0001(4)$ \\
C13 & $0.1011(9)$ & $0.0450(6)$ & $0.0563(6)$ & $0.0149(6)$ & $0.0115(6)$ & $-0.0023(5)$ \\
N4 & $0.0346(15)$ & $0.0383(16)$ & $0.0263(13)$ & $-0.0087(12)$ & $0.0011(11)$ & $-0.0016(11)$ \\
N5 & $0.0377(16)$ & $0.0422(17)$ & $0.0297(13)$ & $-0.0109(13)$ & $0.0008(11)$ & $-0.0011(12)$ \\
C6 & $0.050(2)$ & $0.043(2)$ & $0.0392(19)$ & $-0.0181(18)$ & $-0.0066(16)$ & $-0.0034(16)$ \\
C7 & $0.056(2)$ & $0.043(2)$ & $0.0309(17)$ & $-0.0089(18)$ & $-0.0021(15)$ & $-0.0078(15)$ \\
C8 & $0.0377(18)$ & $0.039(2)$ & $0.0267(15)$ & $-0.0016(15)$ & $-0.0035(13)$ & $-0.0009(14)$ \\
C9 & $0.0342(18)$ & $0.040(2)$ & $0.0279(15)$ & $0.0005(15)$ & $-0.0028(13)$ & $0.0022(14)$ \\
C10 & $0.057(2)$ & $0.045(2)$ & $0.0408(19)$ & $0.0037(19)$ & $0.0075(17)$ & $-0.0028(17)$ \\
C11 & $0.059(3)$ & $0.065(3)$ & $0.046(2)$ & $0.011(2)$ & $0.0181(19)$ & $0.003(2)$ \\
C12 & $0.045(2)$ & $0.076(3)$ & $0.039(2)$ & $-0.006(2)$ & $0.0033(16)$ & $0.017(2)$
\end{tabular}




$\begin{array}{lllllll}\text { C13 } & 0.061(3) & 0.050(2) & 0.040(2) & -0.018(2) & -0.0066(18) & 0.0047(17) \\ \text { C14 } & 0.055(2) & 0.047(2) & 0.0291(16) & -0.0053(18) & 0.0015(15) & 0.0001(15) \\ \text { C15 } & 0.0321(18) & 0.056(2) & 0.0334(17) & -0.0123(17) & 0.0030(13) & 0.0007(16) \\ \text { C16 } & 0.0312(17) & 0.0408(19) & 0.0287(15) & -0.0066(15) & 0.0079(13) & -0.0017(14) \\ \text { N17 } & 0.0319(14) & 0.0340(15) & 0.0284(13) & -0.0035(12) & 0.0016(11) & 0.0011(11) \\ \text { C18 } & 0.0361(18) & 0.0368(19) & 0.0264(15) & -0.0012(15) & 0.0028(13) & -0.0031(13) \\ \text { C19 } & 0.046(2) & 0.050(2) & 0.0329(17) & -0.0017(18) & 0.0001(15) & 0.0083(16) \\ \text { C20 } & 0.058(3) & 0.041(2) & 0.049(2) & -0.0077(19) & 0.0103(18) & 0.0156(17) \\ \text { C21 } & 0.042(2) & 0.047(2) & 0.0427(19) & -0.0185(17) & 0.0074(16) & 0.0024(16) \\ \text { C22 } & 0.0421(19) & 0.0361(19) & 0.0301(16) & -0.0028(15) & -0.0057(14) & -0.0019(13) \\ \text { N23 } & 0.0362(16) & 0.0415(17) & 0.0309(14) & -0.0044(13) & -0.0016(11) & -0.0028(12) \\ \text { C24 } & 0.045(2) & 0.045(2) & 0.0354(18) & -0.0048(17) & 0.0015(15) & -0.0069(16) \\ \text { C25 } & 0.041(2) & 0.043(2) & 0.047(2) & -0.0017(17) & 0.0055(16) & -0.0038(16) \\ \text { C26 } & 0.0341(18) & 0.0339(18) & 0.0375(17) & -0.0085(15) & -0.0001(14) & 0.0033(14) \\ \text { N27 } & 0.0418(17) & 0.0408(17) & 0.0289(14) & -0.0057(14) & -0.0035(12) & 0.0003(12) \\ \text { C28 } & 0.0342(19) & 0.044(2) & 0.0345(17) & -0.0067(16) & 0.0000(14) & 0.0042(15) \\ \text { C29 } & 0.047(2) & 0.052(2) & 0.052(2) & -0.0003(19) & 0.0010(18) & -0.0040(19) \\ \text { C30 } & 0.042(2) & 0.064(3) & 0.059(2) & 0.003(2) & -0.0032(18) & 0.013(2) \\ \text { C31 } & 0.050(2) & 0.073(3) & 0.042(2) & -0.013(2) & -0.0068(18) & 0.004(2) \\ \text { C32 } & 0.067(3) & 0.061(3) & 0.047(2) & -0.004(2) & -0.0086(19) & -0.016(2) \\ \text { C33 } & 0.049(2) & 0.046(2) & 0.052(2) & 0.0038(19) & -0.0074(17) & 0.0010(18) \\ & & & & & & \end{array}$

Geometric parameters $\left(\AA,{ }^{\circ}\right)$

\begin{tabular}{llll}
\hline $\mathrm{Co} 1-\mathrm{N} 4$ & $2.041(3)$ & $\mathrm{C} 18-\mathrm{C} 19$ & $1.381(5)$ \\
$\mathrm{C} 1-\mathrm{N} 17$ & $2.109(3)$ & $\mathrm{C} 18-\mathrm{C} 22$ & $1.515(4)$ \\
$\mathrm{C} 1-\mathrm{C} 13$ & $2.2030(11)$ & $\mathrm{C} 19-\mathrm{C} 20$ & $1.364(5)$ \\
$\mathrm{C} 1-\mathrm{C} 2$ & $2.2499(10)$ & $\mathrm{C} 19-\mathrm{H} 19$ & 0.93 \\
$\mathrm{~N} 4-\mathrm{C} 8$ & $1.354(4)$ & $\mathrm{C} 20-\mathrm{C} 21$ & $1.380(5)$ \\
$\mathrm{N} 4-\mathrm{N} 5$ & $1.361(4)$ & $\mathrm{C} 20-\mathrm{H} 20$ & 0.93 \\
$\mathrm{~N} 5-\mathrm{C} 6$ & $1.341(4)$ & $\mathrm{C} 21-\mathrm{H} 21$ & 0.93 \\
$\mathrm{~N} 5-\mathrm{C} 15$ & $1.459(4)$ & $\mathrm{C} 22-\mathrm{N} 23$ & $1.450(4)$ \\
$\mathrm{C} 6-\mathrm{C} 7$ & $1.362(5)$ & $\mathrm{C} 22-\mathrm{H} 22 \mathrm{~A}$ & 0.97 \\
$\mathrm{C} 6-\mathrm{H} 6$ & 0.93 & $\mathrm{C} 22-\mathrm{H} 22 \mathrm{~B}$ & 0.97 \\
$\mathrm{C} 7-\mathrm{C} 8$ & $1.400(5)$ & $\mathrm{N} 23-\mathrm{C} 24$ & $1.335(4)$ \\
$\mathrm{C} 7-\mathrm{H} 7$ & 0.93 & $\mathrm{~N} 23-\mathrm{N} 27$ & $1.358(3)$ \\
$\mathrm{C} 8-\mathrm{C} 9$ & $1.461(5)$ & $\mathrm{C} 24-\mathrm{C} 25$ & $1.356(5)$ \\
$\mathrm{C} 9-\mathrm{C} 14$ & $1.386(5)$ & $\mathrm{C} 24-\mathrm{H} 24$ & 0.93 \\
$\mathrm{C} 9-\mathrm{C} 10$ & $1.390(5)$ & $\mathrm{C} 25-\mathrm{C} 26$ & $1.395(5)$ \\
$\mathrm{C} 10-\mathrm{C} 11$ & $1.378(5)$ & $\mathrm{C} 25-\mathrm{H} 25$ & 0.93 \\
$\mathrm{C} 10-\mathrm{H} 10$ & 0.93 & $\mathrm{C} 26-\mathrm{N} 27$ & $1.331(4)$ \\
$\mathrm{C} 11-\mathrm{C} 12$ & $1.376(6)$ & $\mathrm{C} 26-\mathrm{C} 28$ & $1.482(4)$ \\
$\mathrm{C} 11-\mathrm{H} 11$ & 0.93 & $\mathrm{C} 28-\mathrm{C} 33$ & $1.375(5)$ \\
$\mathrm{C} 12-\mathrm{C} 13$ & $1.366(6)$ & $\mathrm{C} 28-\mathrm{C} 29$ & $1.388(5)$ \\
$\mathrm{C} 12-\mathrm{H} 12$ & 0.93 & $\mathrm{C} 29-\mathrm{C} 30$ & $1.393(5)$ \\
$\mathrm{C} 13-\mathrm{C} 14$ & $1.383(5)$ & $\mathrm{C} 29-\mathrm{H} 29$ & 0.93 \\
$\mathrm{C} 13-\mathrm{H} 13$ & 0.93 & $\mathrm{C} 30-\mathrm{C} 31$ & $1.356(6)$ \\
$\mathrm{C} 14-\mathrm{H} 14$ & 0.93 & $\mathrm{C} 30-\mathrm{H} 30$ & 0.93
\end{tabular}




\begin{tabular}{|c|c|c|c|}
\hline $\mathrm{C} 15-\mathrm{C} 16$ & $1.510(4)$ & $\mathrm{C} 31-\mathrm{C} 32$ & $1.375(6)$ \\
\hline $\mathrm{C} 15-\mathrm{H} 15 \mathrm{~A}$ & 0.97 & $\mathrm{C} 31-\mathrm{H} 31$ & 0.93 \\
\hline $\mathrm{C} 15-\mathrm{H} 15 \mathrm{~B}$ & 0.97 & $\mathrm{C} 32-\mathrm{C} 33$ & $1.390(5)$ \\
\hline $\mathrm{C} 16-\mathrm{N} 17$ & $1.361(4)$ & $\mathrm{C} 32-\mathrm{H} 32$ & 0.93 \\
\hline $\mathrm{C} 16-\mathrm{C} 21$ & $1.369(5)$ & $\mathrm{C} 33-\mathrm{H} 33$ & 0.93 \\
\hline $\mathrm{N} 17-\mathrm{C} 18$ & $1.349(4)$ & & \\
\hline $\mathrm{N} 4-\mathrm{Co} 1-\mathrm{N} 17$ & $96.51(10)$ & $\mathrm{C} 16-\mathrm{N} 17-\mathrm{Co} 1$ & $116.9(2)$ \\
\hline $\mathrm{N} 4-\mathrm{Co} 1-\mathrm{Cl} 3$ & $118.60(9)$ & $\mathrm{N} 17-\mathrm{C} 18-\mathrm{C} 19$ & $121.9(3)$ \\
\hline $\mathrm{N} 17-\mathrm{Co} 1-\mathrm{Cl} 3$ & $108.05(8)$ & $\mathrm{N} 17-\mathrm{C} 18-\mathrm{C} 22$ & $117.4(3)$ \\
\hline $\mathrm{N} 4-\mathrm{Co} 1-\mathrm{Cl} 2$ & $109.65(8)$ & $\mathrm{C} 19-\mathrm{C} 18-\mathrm{C} 22$ & $120.6(3)$ \\
\hline $\mathrm{N} 17-\mathrm{Co} 1-\mathrm{Cl} 2$ & $108.85(8)$ & $\mathrm{C} 20-\mathrm{C} 19-\mathrm{C} 18$ & $119.8(3)$ \\
\hline $\mathrm{Cl} 3-\mathrm{Co} 1-\mathrm{Cl} 2$ & $113.51(5)$ & $\mathrm{C} 20-\mathrm{C} 19-\mathrm{H} 19$ & 120.1 \\
\hline $\mathrm{C} 8-\mathrm{N} 4-\mathrm{N} 5$ & $105.7(2)$ & $\mathrm{C} 18-\mathrm{C} 19-\mathrm{H} 19$ & 120.1 \\
\hline $\mathrm{C} 8-\mathrm{N} 4-\mathrm{Co} 1$ & $136.3(2)$ & $\mathrm{C} 19-\mathrm{C} 20-\mathrm{C} 21$ & $119.0(3)$ \\
\hline $\mathrm{N} 5-\mathrm{N} 4-\mathrm{Co} 1$ & $115.88(18)$ & $\mathrm{C} 19-\mathrm{C} 20-\mathrm{H} 20$ & 120.5 \\
\hline $\mathrm{C} 6-\mathrm{N} 5-\mathrm{N} 4$ & $111.2(3)$ & $\mathrm{C} 21-\mathrm{C} 20-\mathrm{H} 20$ & 120.5 \\
\hline $\mathrm{C} 6-\mathrm{N} 5-\mathrm{C} 15$ & $129.2(3)$ & $\mathrm{C} 16-\mathrm{C} 21-\mathrm{C} 20$ & $119.1(3)$ \\
\hline $\mathrm{N} 4-\mathrm{N} 5-\mathrm{C} 15$ & $119.5(3)$ & $\mathrm{C} 16-\mathrm{C} 21-\mathrm{H} 21$ & 120.5 \\
\hline $\mathrm{N} 5-\mathrm{C} 6-\mathrm{C} 7$ & $107.5(3)$ & $\mathrm{C} 20-\mathrm{C} 21-\mathrm{H} 21$ & 120.5 \\
\hline $\mathrm{N} 5-\mathrm{C} 6-\mathrm{H} 6$ & 126.3 & $\mathrm{~N} 23-\mathrm{C} 22-\mathrm{C} 18$ & $112.4(3)$ \\
\hline $\mathrm{C} 7-\mathrm{C} 6-\mathrm{H} 6$ & 126.3 & $\mathrm{~N} 23-\mathrm{C} 22-\mathrm{H} 22 \mathrm{~A}$ & 109.1 \\
\hline $\mathrm{C} 6-\mathrm{C} 7-\mathrm{C} 8$ & $106.5(3)$ & $\mathrm{C} 18-\mathrm{C} 22-\mathrm{H} 22 \mathrm{~A}$ & 109.1 \\
\hline $\mathrm{C} 6-\mathrm{C} 7-\mathrm{H} 7$ & 126.7 & $\mathrm{~N} 23-\mathrm{C} 22-\mathrm{H} 22 \mathrm{~B}$ & 109.1 \\
\hline $\mathrm{C} 8-\mathrm{C} 7-\mathrm{H} 7$ & 126.7 & $\mathrm{C} 18-\mathrm{C} 22-\mathrm{H} 22 \mathrm{~B}$ & 109.1 \\
\hline $\mathrm{N} 4-\mathrm{C} 8-\mathrm{C} 7$ & $109.1(3)$ & $\mathrm{H} 22 \mathrm{~A}-\mathrm{C} 22-\mathrm{H} 22 \mathrm{~B}$ & 107.9 \\
\hline $\mathrm{N} 4-\mathrm{C} 8-\mathrm{C} 9$ & $123.4(3)$ & $\mathrm{C} 24-\mathrm{N} 23-\mathrm{N} 27$ & $112.0(3)$ \\
\hline $\mathrm{C} 7-\mathrm{C} 8-\mathrm{C} 9$ & $127.5(3)$ & $\mathrm{C} 24-\mathrm{N} 23-\mathrm{C} 22$ & $127.5(3)$ \\
\hline $\mathrm{C} 14-\mathrm{C} 9-\mathrm{C} 10$ & $118.0(3)$ & $\mathrm{N} 27-\mathrm{N} 23-\mathrm{C} 22$ & $120.4(3)$ \\
\hline $\mathrm{C} 14-\mathrm{C} 9-\mathrm{C} 8$ & $123.0(3)$ & $\mathrm{N} 23-\mathrm{C} 24-\mathrm{C} 25$ & $107.3(3)$ \\
\hline $\mathrm{C} 10-\mathrm{C} 9-\mathrm{C} 8$ & $118.9(3)$ & $\mathrm{N} 23-\mathrm{C} 24-\mathrm{H} 24$ & 126.3 \\
\hline $\mathrm{C} 11-\mathrm{C} 10-\mathrm{C} 9$ & $121.2(4)$ & $\mathrm{C} 25-\mathrm{C} 24-\mathrm{H} 24$ & 126.3 \\
\hline $\mathrm{C} 11-\mathrm{C} 10-\mathrm{H} 10$ & 119.4 & $\mathrm{C} 24-\mathrm{C} 25-\mathrm{C} 26$ & $105.3(3)$ \\
\hline $\mathrm{C} 9-\mathrm{C} 10-\mathrm{H} 10$ & 119.4 & $\mathrm{C} 24-\mathrm{C} 25-\mathrm{H} 25$ & 127.4 \\
\hline $\mathrm{C} 12-\mathrm{C} 11-\mathrm{C} 10$ & $119.7(4)$ & $\mathrm{C} 26-\mathrm{C} 25-\mathrm{H} 25$ & 127.4 \\
\hline $\mathrm{C} 12-\mathrm{C} 11-\mathrm{H} 11$ & 120.1 & $\mathrm{~N} 27-\mathrm{C} 26-\mathrm{C} 25$ & $111.1(3)$ \\
\hline $\mathrm{C} 10-\mathrm{C} 11-\mathrm{H} 11$ & 120.1 & $\mathrm{~N} 27-\mathrm{C} 26-\mathrm{C} 28$ & $121.2(3)$ \\
\hline $\mathrm{C} 13-\mathrm{C} 12-\mathrm{C} 11$ & $119.8(4)$ & $\mathrm{C} 25-\mathrm{C} 26-\mathrm{C} 28$ & $127.5(3)$ \\
\hline $\mathrm{C} 13-\mathrm{C} 12-\mathrm{H} 12$ & 120.1 & $\mathrm{C} 26-\mathrm{N} 27-\mathrm{N} 23$ & $104.2(3)$ \\
\hline $\mathrm{C} 11-\mathrm{C} 12-\mathrm{H} 12$ & 120.1 & $\mathrm{C} 33-\mathrm{C} 28-\mathrm{C} 29$ & $118.6(3)$ \\
\hline $\mathrm{C} 12-\mathrm{C} 13-\mathrm{C} 14$ & $120.7(4)$ & $\mathrm{C} 33-\mathrm{C} 28-\mathrm{C} 26$ & $121.2(3)$ \\
\hline $\mathrm{C} 12-\mathrm{C} 13-\mathrm{H} 13$ & 119.7 & $\mathrm{C} 29-\mathrm{C} 28-\mathrm{C} 26$ & $120.2(3)$ \\
\hline $\mathrm{C} 14-\mathrm{C} 13-\mathrm{H} 13$ & 119.7 & $\mathrm{C} 28-\mathrm{C} 29-\mathrm{C} 30$ & $120.4(4)$ \\
\hline $\mathrm{C} 13-\mathrm{C} 14-\mathrm{C} 9$ & $120.4(3)$ & $\mathrm{C} 28-\mathrm{C} 29-\mathrm{H} 29$ & 119.8 \\
\hline $\mathrm{C} 13-\mathrm{C} 14-\mathrm{H} 14$ & 119.8 & $\mathrm{C} 30-\mathrm{C} 29-\mathrm{H} 29$ & 119.8 \\
\hline $\mathrm{C} 9-\mathrm{C} 14-\mathrm{H} 14$ & 119.8 & $\mathrm{C} 31-\mathrm{C} 30-\mathrm{C} 29$ & $120.4(4)$ \\
\hline $\mathrm{N} 5-\mathrm{C} 15-\mathrm{C} 16$ & $112.8(3)$ & $\mathrm{C} 31-\mathrm{C} 30-\mathrm{H} 30$ & 119.8 \\
\hline $\mathrm{N} 5-\mathrm{C} 15-\mathrm{H} 15 \mathrm{~A}$ & 109 & $\mathrm{C} 29-\mathrm{C} 30-\mathrm{H} 30$ & 119.8 \\
\hline
\end{tabular}




\begin{tabular}{llll}
$\mathrm{C} 16-\mathrm{C} 15-\mathrm{H} 15 \mathrm{~A}$ & 109 & $\mathrm{C} 30-\mathrm{C} 31-\mathrm{C} 32$ & $119.9(4)$ \\
$\mathrm{N} 5-\mathrm{C} 15-\mathrm{H} 15 \mathrm{~B}$ & 109 & $\mathrm{C} 30-\mathrm{C} 31-\mathrm{H} 31$ & 120 \\
$\mathrm{C} 16-\mathrm{C} 15-\mathrm{H} 15 \mathrm{~B}$ & 109 & $\mathrm{C} 32-\mathrm{C} 31-\mathrm{H} 31$ & 120 \\
$\mathrm{H} 15 \mathrm{~A}-\mathrm{C} 15-\mathrm{H} 15 \mathrm{~B}$ & 107.8 & $\mathrm{C} 31-\mathrm{C} 32-\mathrm{C} 33$ & $120.2(4)$ \\
$\mathrm{N} 17-\mathrm{C} 16-\mathrm{C} 21$ & $122.5(3)$ & $\mathrm{C} 31-\mathrm{C} 32-\mathrm{H} 32$ & 119.9 \\
$\mathrm{~N} 17-\mathrm{C} 16-\mathrm{C} 15$ & $115.6(3)$ & $\mathrm{C} 33-\mathrm{C} 32-\mathrm{H} 32$ & 119.9 \\
$\mathrm{C} 21-\mathrm{C} 16-\mathrm{C} 15$ & $121.8(3)$ & $\mathrm{C} 28-\mathrm{C} 33-\mathrm{C} 32$ & 119.7 \\
$\mathrm{C} 18-\mathrm{N} 17-\mathrm{C} 16$ & $117.4(3)$ & $\mathrm{C} 28-\mathrm{C} 33-\mathrm{H} 33$ & 119.7 \\
$\mathrm{C} 18-\mathrm{N} 17-\mathrm{C} 1$ & $124.2(2)$ & $\mathrm{C} 32-\mathrm{C} 33-\mathrm{H} 33$ & 19.7 \\
\hline
\end{tabular}

Hydrogen-bond geometry $\left(\AA,{ }^{\circ}\right)$

\begin{tabular}{lllll}
\hline$D-\mathrm{H} \cdots A$ & $D-\mathrm{H}$ & $\mathrm{H} \cdots A$ & $D \cdots A$ & $D-\mathrm{H} \cdots A$ \\
\hline $\mathrm{C} 11-\mathrm{H} 11 \cdots C g 1^{\mathrm{i}}$ & 0.93 & 2.87 & $3.806(4)$ & 180 \\
\hline
\end{tabular}

Symmetry code: (i) $-x+1,-y,-z+1$. 